\title{
Tunable polarization components and electric field induced crystallization in polyvinylidenefluoride: A piezo polymer
}

\author{
Ronit Ganguly $^{1}$ | Soumya Bandyopadhyay ${ }^{2}$ | Kumaraswamy Miriyala ${ }^{2}$ | \\ Vijayabhaskar Gunasekaran ${ }^{1}$ | Saswata Bhattacharya ${ }^{2}$ | Amit Acharyya ${ }^{1}$ | \\ Ranjith Ramadurai ${ }^{2}$
}

${ }^{1}$ Department of Electrical Engineering, Indian Institute of Technology Hyderabad, Sangareddy, Telangana, India

${ }^{2}$ Department of Materials Science and Metallurgical Engineering, Indian Institute of Technology Hyderabad, Sangareddy,

Telangana, India

\section{Correspondence}

Ranjith Ramadurai, Department of Materials Science and Metallurgical Engineering, Indian Institute of Technology Hyderabad, Sangareddy 502285, Telangana, India. Email: ranjith@iith.ac.in

\begin{abstract}
Polyvinylidenefluoride (PVDF) a semicrystalline pieozoelectric polymer was synthesized with varying process conditions and its ferroelectric domain orientations were studied using piezoresponse force microscope (PFM). PVDF thin films fabricated using tape casting technique with precursor solutions of varying viscosities reveal that the polarization components transform from a dominant planar to an out-of-plane configuration with increase in viscosity. Interestingly the planar components possessed a head to head or tail to tail kind of paired domains separated by a distance of $\sim 380-400 \mathrm{~nm}$. Electrostatic energy minimization of an electrically inhomogeneous system containing similar domain arrangements as the experiments shows that the head to head and tail to tail arrangements with a minimum separation distance are more favorable than head to tail arrangements of domains. With increment of applied field, the domains grew in size and shape indicating amorphous to crystalline transformation of PVDF films. Such transformation was evident from X-ray diffraction studies performed in-situ in the presence of an applied electric field.
\end{abstract}

KEYWORDS

PVDF, Ferroelectriciy, Piezoelectricity, Polarization, Crystallinity

\section{1 | INTRODUCTION}

Piezoelectric polymers and hybrid nanocomposites comprising piezo polymers are gaining attention as smart materials in various applications, such as sensors, actuators, wearable energy harvesters and flexible electronic devices. ${ }^{[1-4]}$ Amid the few available piezo-polymers, poly(vinylidenefluoride) (PVDF) and its copolymers are most widely used due to their improved thermal stability, chemical resistance, and mechanical strength. PVDF is a semi-crystalline polymer possessing various crystalline phases. There have been ample studies on PVDF, its copolymers and nanocomposites, focussing the structure and multifunctional property. ${ }^{[5-7]}$ PVDF is known to stabilize in five distinct crystalline phases related to different chain conformations $\alpha, \beta, \gamma, \delta$, and $\varepsilon$, in which the most investigated phases are $\alpha, \beta, \gamma \cdot{ }^{[5,8-11]} \beta$-phase commonly known as phase I exhibits superior piezoelectric properties due to its non-centrosymmetric crystalline state with the dipole moment of two chains containing $\mathrm{C}-\mathrm{F}$ and $\mathrm{C}-\mathrm{H}$ in the unit cell adding up resulting in a net dipole moment perpendicular to the carbon backbone with fluorine and hydrogen as the negative and positive poles, respectively. ${ }^{[6,12-14]}$

PVDF thin films and its properties are known to be sensitive to the methods adapted for fabrication. ${ }^{[5,15,16]}$ Literature suggests that the transformation among the different phases of PVDF occurs in a given sequence $\alpha \rightarrow \gamma \rightarrow \beta$ with varying temperature and/or strain induced processing. ${ }^{[10,17-19]}$ However, PVDF films results in mixed phase configuration when prepared under normal tape casting methods. PVDF films prepared under specific conditions like tensile stress, electric field, co-polymerization etc., results into homogenous single phase material. ${ }^{[5,13,16]}$ Electrospinning is another technique widely used to fabricate nano wire/fabric samples. ${ }^{[5]}$ Though every synthesis techniques have their unique process conditions, major characteristics like, crystalline configuration, morphology, porosity, optical transparency, and mechanical properties of PVDF films synthesized through solution casting technique is dependent on the viscosity of the initial solution. ${ }^{[20-22]}$ 
Though the macroscopic ferroelectric characteristics of PVDF has been studied and utilized in applications, ${ }^{[23,24]}$ there have been limited studies on the formation of nanoscale ferroelectric domains and switching behavior in PVDF. The earlier studies, ${ }^{[23]}$ on completely crystalline PVDF- co-polymers show signatures of ferroelectric domain with specific polarization orientation adding on to the lamellar behavior of the crystalline phases. ${ }^{[25,26]}$ This lamella that forms the basis of the crystalline region of PVDF is known to possess the ferroelectric domains. The net polarization and its switching is expected to arise due to the rotation of molecules around the chain. Hence, the electrostatic nature of ferroelectric domain arises due to arrangement of the chain in a particular configuration. There are studies that suggest that charged domain wall exists in ferroelectric ceramic because of head-to-head or tail-to-tail arrangements of domain. ${ }^{[27]}$ The electrostatic strength of domains could possibly have influence on the adjacent ferroelectric domains in certain region. In PVDF and its co-polymers, external electric field has a significant influence on the structural transitions. ${ }^{[28-31]}$ Electric poling induced crystallinity has been studied earlier through $x$-ray diffraction studies. ${ }^{[32-34]}$ Poling are usually performed at high electric field where as not much attention has been given to low electric field studies. Studies that involves low electric field during fabrication of PVDF fibers like electrospinning has emphasized on the piezoelectric and ferroelectric property of nano fabric/wire. ${ }^{[35-37]}$ Further enhancement of $d_{33}$ upto $63 \mathrm{pm} / \mathrm{V}$ was observed when composite PVDF fibers were prepared by electrospinning, however the details of domain pattern remains unclear. ${ }^{[37]}$ Crystalline transformation associated with low electric field provides an insight into the ferroelectric domains and their piezoresponse behaviors.

In this work we address the tunability of polarization components offered by the preparation conditions of semi-crystalline pure PVDF film. The polarization components of the films were investigated using PFM and computation based on electrostatic energy minimization was carried out to understand the formation and stability of the FE domains and their transformation under applied electric field. The experimental observations of domain separation distance were validated using the numerical solutions of Poisson's equation for electrically inhomogeneous systems. The plausible enhancement of crystallinity in the presence of electric field was studied by performing $x$-ray diffraction (XRD) studies in the presence of the electric field.

\section{2 | EXPERIMENTAL SECTION}

\section{1 | Materials and sample preparation by solution casting method}

Commercially available PVDF (Sigma Aldrich, St. Louis, Missouri, USA) powders and N-Methyl-2-pyrrolidone (NMP) (Sigma Aldrich, St. Louis, Missouri, USA) solvent with appropriate weight proportion were mixed in a magnetic stirrer to obtain the solution of PVDF. The stirring was performed at $60^{\circ} \mathrm{C}$ temperature for 90 minutes. The PVDF solutions with varying viscosities were prepared by varying the weight percentage of PVDF powder. PVDF solution with varying viscosity range 2-8 Pa.S was synthesized and further utilized for the fabrication of thin films. The solutions were casted to films on Aluminium foil/borosilicate microslide glass by the conventional doctor Blade technique and further baked at $60^{\circ} \mathrm{C}$ for
8 hours. Even though baking temperature is enough to produce $\beta$ phase, in order to attain higher content, the thin films were annealed at $90^{\circ} \mathrm{C}$.

\section{2 | Characterizations}

The phase identification of the fabricated films was studied by using X-ray diffractometer (Bruker D8 Discover, Billerica, Massachusetts, USA) with $\mathrm{Cu} \mathrm{K} \alpha$ radiation. A well separated (Pt) electrode pattern that could apply a planar field was deposited on PVDF films and utmost care was taken to make sure the X-ray beam falls on the polymer film between the electrodes for the electric field induced crystallinity studies. The vibrational modes of the fabricated PVDF films were detected using Raman (Bruker SENTERRA dispersive micro Raman spectrometer, Billerica, Massachusetts, USA) and Fouriertransform infrared spectroscopy (FTIR) (Bruker ALPHA-T, Billerica, Massachusetts, USA) studies. The viscosity of the PVDF solutions were studied using a Rheometer (Physica MCR 301, Anton Paar $\mathrm{GmbH}$, Anton-Paar-Str. 20, A-8054 Graz, Austria) at shear rate of $100 / \mathrm{s}$. The morphological studies of the films were carried by SEM (JEOL JSM 7800F, Akishima, Tokyo 196-8558, Japan). Further Piezoresponse force microscopy studies were carried out using the Nanoscope V controller (make; Bruker, Billerica, Massachusetts, USA Model: Dimension ICON).Commercially available $\mathrm{Co} / \mathrm{Cr}$ (Spring constant $=2.8$ $\mathrm{N} / \mathrm{m}$; Resonant frequency $=75 \mathrm{KHz}$ ) coated tips were used for mapping the IP and OP PFM images. The IP and OP amplitude and Phase images were obtained by applying an Ac drive amplitude of $20-25 \mathrm{mV}$ at $15 \mathrm{KHz}$. A Dc bias of $5 \mathrm{~V}$ and $10 \mathrm{~V}$ was subjected to the tip along with the $\mathrm{AC}$ signal of $5 \mathrm{~V}$ amplitude for inducing the electric field over the sample surface to observe the domain growth.

\section{3 | RESULTS AND DISCUSSION}

\section{1 | Crystalline phase identification}

In this study we systematically vary the viscosity of precursor solution. The variation in viscosity plays an important role in our observation. The viscosities are 2.4, 2.9, 4.6, 8.1 Pa.s respectively. The thin films prepared by precursor solution of these viscosities are correspondingly labeled as PVDF 2.4, PVDF 2.9, PVDF 4.6, PVDF 8.1. Here onwards we will be using these labels to refer to films made from particular precursor viscosity. In this study we have used aluminium foil and microslide glass substrates to fabricate PVDF thin films. However, all the samples that were fabricated on aluminium foil was preferred due to the bottom electrode requirement for PFM studies. Studies have has shown that aluminium substrate enhances the effective sensitivity (in terms of $d_{33}$ ) which also indicate toward in enhancement of electroactive phase ( $\beta / \gamma$ - phases). ${ }^{[38]}$ On comparing our results of aluminium and glass substrate based PVDF we observe that in both the samples $\beta$-phase was dominating. Hence in this study to avoid the influence of substrate surface all the samples were fabricated on the aluminium substrate obtained from the same larger sheet of aluminium with no significant change in morphology between the substrates chosen for fabrication. In addition, polymer-solvent interaction plays a vital role to determine the morphology of films. ${ }^{[20,21]}$ Studies show 
that baking temperature has an influence on the pore formation in PVDF thin films. ${ }^{[39]}$ Higher drying temperatures lead to higher solvent evaporation rates, which in turn avoid the formation of pores, thus resulting in low porous samples. ${ }^{[40,41]}$ High porosity is attributed to the polymer chains mobility which reduces with decreasing temperature and prevents the polymer chains to occupy the free space left behind by the evaporated solvent. It is known that higher the porosity leads to higher roughness thus influencing the appearance of the film. ${ }^{[22]}$ From scanning electron microscopy(SEM) studies (not shown) we found out that with increase in viscosity the pore size and the distribution of pores in $100 \mu \mathrm{m} \times 100 \mu \mathrm{m}$ decreases significantly. PFM studies were performed at nanoscale regions such that the porosity does not interfere with the imaging of domain pattern. The inset of Figure $1 \mathrm{~A}$ shows the X-ray diffraction pattern of PVDF 4.6 sample. The peak at $2 \theta=20.2^{\circ}$ relative to the sum of diffraction from the planes (200) and (110) confirms the presence of $\beta$-phase in PVDF films and the shoulder peak at $18.5^{\circ}$ corresponding to (020) plane indicates the existence of a minute amount of $\gamma$-phase. ${ }^{[5,9,17,42-44]}$ The films prepared from varying viscosities seem to have no change in the percentage of mixed phases present, however a minor enhancement in the crystallinity of PVDF films was observed when synthesized from higher viscous solutions.

Figure 1A shows the Raman spectra of PVDF 4.6 sample. It is observed that the spectra are dominated by a band at $839 \mathrm{~cm}^{-1}$. The high intensity peak at $839 \mathrm{~cm}^{-1}$ confirms the high percentage $\beta$-phase for annealed films. The Raman bands at $811 \mathrm{~cm}^{-1}$ in PVDF films, corresponds to $\gamma$ phase and the peak at $882 \mathrm{~cm}^{-1}$ corresponds to the mixed phases. ${ }^{[18]}$ It is known that the solution casting method often gives rise to mixed phases which is also evident from our results. As our interest is mainly on understanding the ferroelectric domains of pure PVDF and its semi-crystalline nature we continued our studies with PVDF and not sticking to the methods known to enhance the $\beta$-phase. ${ }^{[5,14,44,45]}$ The above studies confirm that the PVDF film mainly consists of $\beta$-phase along with minor fraction of other $\gamma$ phase. Figure 1B shows the FTIR spectrum of PVDF 4.6 sample in absorption mode. The intensity peak at $833 \mathrm{~cm}^{-1}, 875 \mathrm{~cm}^{-1}$ confirms the presence of $\gamma$ and $\beta$ phase. The peak at $1070 \mathrm{~cm}^{-1}$ corresponds to mixed phase. The phase fraction of $\gamma$ to $\beta$ phase was found to be 0.86 . It is evident that the $\beta$ phase is predominant in the film. On a comparative observation of the results from these methods it can be concluded that overall crystallinity of $\beta$ phase fraction ranges from $80 \%$ to $85 \%$ which is in correlation with the Raman scattering studies.

\section{2 | Piezoresponse force microscopy}

The PFM studies of the piezoelectrics and ferroelectrics include domain imaging, polarization switching and local spectroscopic studies. In this method a ferroelectric sample is placed between the bottom electrode and the PFM tip, which act as a top electrode during the measurements. We carried out measurements in the contact mode to gather surface response information normal (out-of-plane component) and parallel to the film plane (in-plane component) via the frictional forces. ${ }^{[46]}$ The PFM scan give images of morphology, amplitude, and phase which corresponds to topography, piezoelectric coefficient $\left(d_{33}\right)$ and the orientations of the polarization field relative to the plane of the films. An application of the positive or negative dc bias to the tip can induce $180^{\circ}$ polarization switching, orienting polarization upward or downward. ${ }^{[47]}$

Figure $2 \mathrm{~A}-\mathrm{H}$ shows the in-plane and out-of-plane PFM amplitude of four different viscosities. Piezoresponse amplitude values for every sample were determined by averaging amplitude values from 25 different position in the scan area. Figure $3 \mathrm{~A}$ shows the variation of inplane piezoresponse amplitudes with varying viscosity. With the increase in viscosity, the in-plane response of the sample increases up to a viscosity of 4.6 Pa-s and decreases thereafter. This is also evident from the Figure $2 \mathrm{C}$ of PVDF 4.6 sample where the contrasts in PFM amplitude from polar domains are more prominent in comparison to surrounding nonpolar matrix. Out-of-plane polarization component from PFM amplitude images shows a weaker contrast. The $d_{33}$ of the film is calculated using the out-of-plane components. Figure $3 B$ shows the variation of $d_{33}$ with varying viscosity. It was observed that PVDF 2.9 has the highest $d_{33}$, which indicates that it has the highest out-ofplane polarization component. With increasing viscosity there is a weakening in out-of-plane components and the $d_{33}$ decreases.

It is evident from the above observations that the in-plane and out-of-plane polarization components exhibit different behavioral pattern with the variation in viscosity. In our PVDF films the in-plane domains are dominant for the entire range of viscosities. There is a pronounced increase in the out-of-plane domains for lower viscosities and there after we see increase in in-plane domain until an optimum viscosity is reached. The optimum viscosity of in-plane polarization is
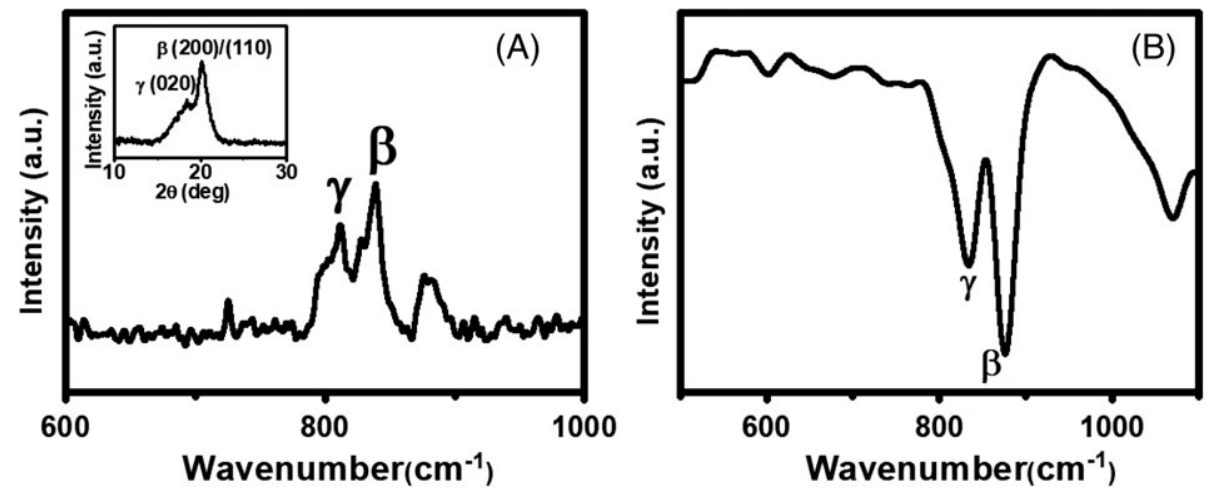

FIGURE 1 A, X-ray diffraction (XRD) and Raman spectra of polyvinylidenefluoride (PVDF) film. B, Fourier-transform infrared spectroscopy (FTIR) spectra of PVDF film 
PVDF 2.4

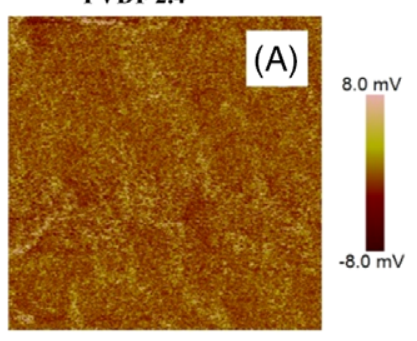

$0 \quad$ IP-Amplitude $10 \mu \mathrm{m}$

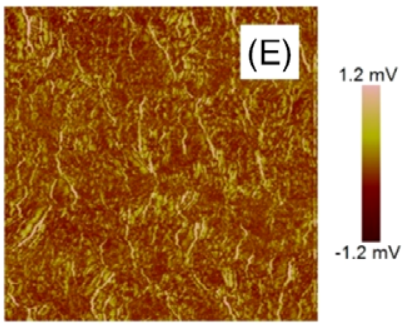

0 OP-Amplitude $10 \mu \mathrm{m}$
PVDF 2.9

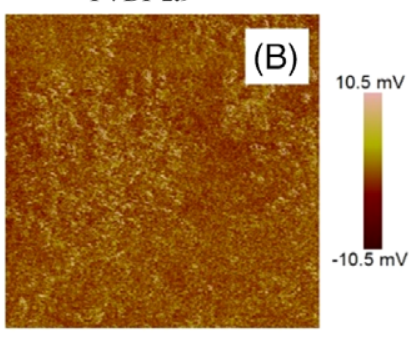

$0 \quad$ IP-Amplitude $10 \mu \mathrm{m}$

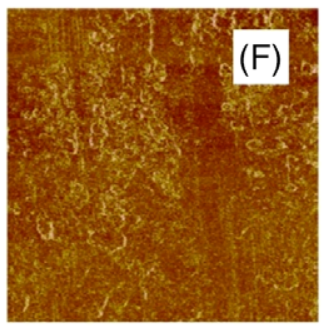

0 OP-Amplitude $10 \mu \mathrm{m}$
PVDF 4.6

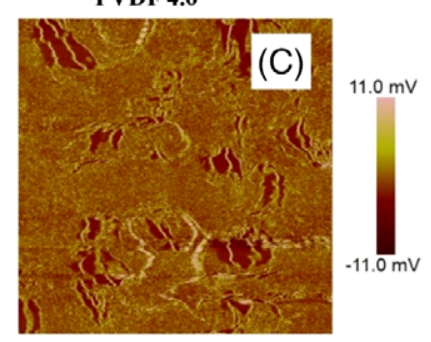

0 IP-Amplitude $10 \mu \mathrm{m}$

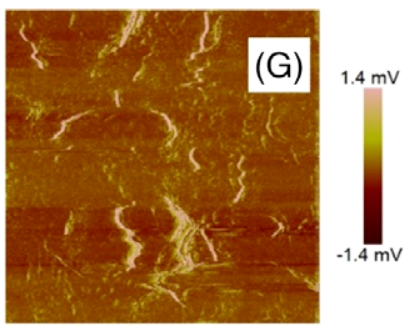

$0 \quad$ OP-Amplitude $10 \mu \mathrm{m}$
PVDF 8.1

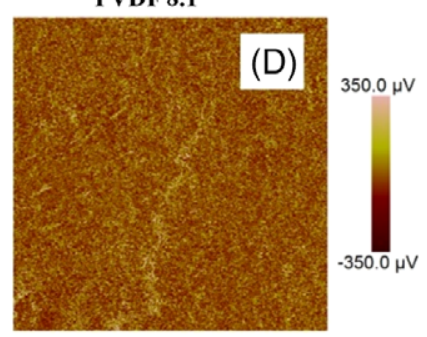

0 IP-Amplitude $10 \mu \mathrm{m}$

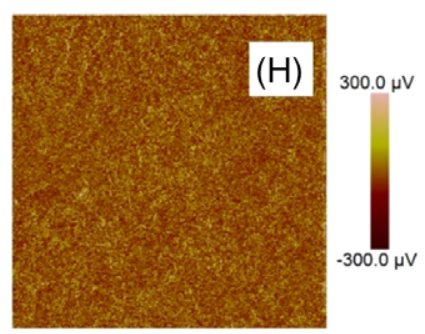

0 OP-Amplitude $10 \mu \mathrm{m}$

FIGURE 2 (A-D), in-plane and (E-H), out of plane PFM amplitude scans of different samples

4.6 Pa.s and out-of-plane polarization is 2.9 Pa.s. The variation in polarization component can be attributed to the crystalline nature of PVDF which in turn is sensitive to the viscosity of precursor solution. ${ }^{[48]}$ Viscosities of melt has an influence on crystallization conditions which in turn effects crystalline structures. Hence viscosity plays a vital role in deciding the crystallinity (chain structure) of a polymer. ${ }^{[48,49]}$ Higher viscosities results in reduction of both in-plane and out-of-plane domains. This reduction might be due to reason that polymeric chains of crystalline and amorphous phase entangle each other that hinders any movement or rotation of monomers.

PFM studies reveal the presence of various polarization components in which, the piezoresponse amplitude and phase image of the respective films gives better understanding of the ferroelectric domain patterns present. Figure 4 represents the PFM images of PVDF 4.6 sample. Various features like morphology, out-of-plane (OP) and inplane (IP) amplitude, phase images were shown in Figure 4A-F. Interestingly the Figure $4 \mathrm{E}$ reveals the presence of dominant IP polarization components in this particular film. It evidently shows the presence of paired regions with opposite domain orientations either left or right with respect to the probe tip. The domains are out of phase to each other by $180^{\circ}$ and are separated by a distance of 380-400 nm. This distance observed is relatively larger in comparison to conventional ferroelectric domain separations. However,
(A)

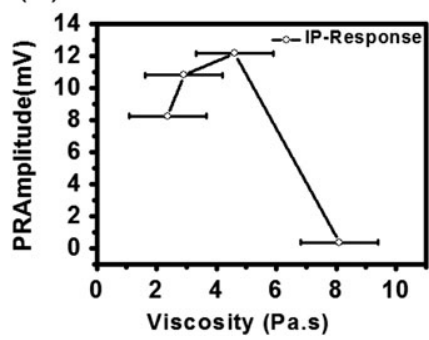

(B)

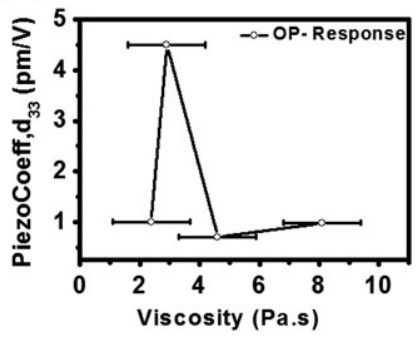

FIGURE 3 A, Graph showing in-plane response for different viscosities $B$, graph showing $d_{33}$ coefficient for different viscosities conventionally the ferroelectric domain walls are $180^{\circ}$ with either head-to-tail or tail-to-head configuration. In this case we believe that the intermediate region presumably amorphous is capable of storing excess charge. ${ }^{[50-52]}$ Hence, maintaining a distance of few $100 \mathrm{~nm}$ is necessary to stabilize the head to head or tail to tail configurations.

As these planar domains are of similar to same charges facing each other, it results in an electrostatic interaction within the pair of domains. Hence, electrostatic force from domains is expected to influence the nucleation of other domain in the surrounding area. It is evident from Figure $4 \mathrm{~F}$ that a criss-cross configuration of domain orientation was observed between the adjacent pair of domains. The arrow marks are representative guide for the eyes and an opposite representation with $180^{\circ}$ rotation is equally plausible.

The PFM scan are said to be sensitive to surface roughness and have higher chances of topographic cross talk. In order to confirm that our results are not surface dominated we did a study on the morphology influence on the domain pattern. We have highlighted ferroelectric domains and corresponding areas in morphology image as shown in Figure 5A. We have observed the domain patterns are evolving from a thick, bright uniform region. Moreover we do see some rough regions in the morphology and the corresponding phase image is not showing any color contrast in those regions. A closer look to the domain was also performed as seen in Figure $5 B$, it was observe that there was no change in height with respect to the morphological region corresponding to occurrence of domain. This implies that the observed domain pattern is independent from its morphological influence. Further the topographic cross talk takes place if we operate the tip close to its contact resonance frequency (typically few hundreds of $\mathrm{kHz}$ ). To avoid such topographic influence we have operated the tip at a frequency (operated frequency: $15 \mathrm{kHz}$ ) far from its resonant frequency. Large area scans show the presence of uniformly distributed paired domains were found to be distributed uniformly throughout the sample and the scalable nature of the domain patterns is evident (Figure 6). 

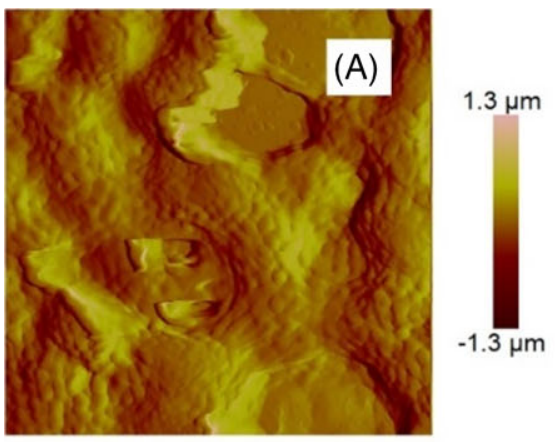

$\mathbf{0}$ Morphology

$10 \mu \mathrm{m}$

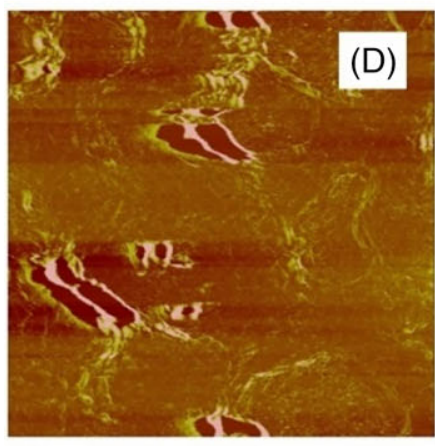

0 IP-Amplitude

$10 \mu \mathrm{m}$
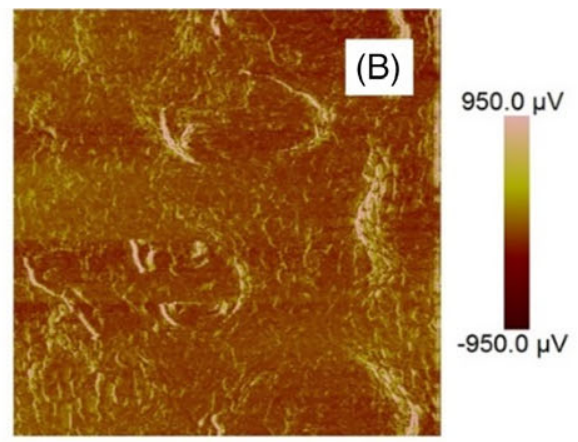

0

OP-Amplitude

$10 \mu \mathrm{m}$

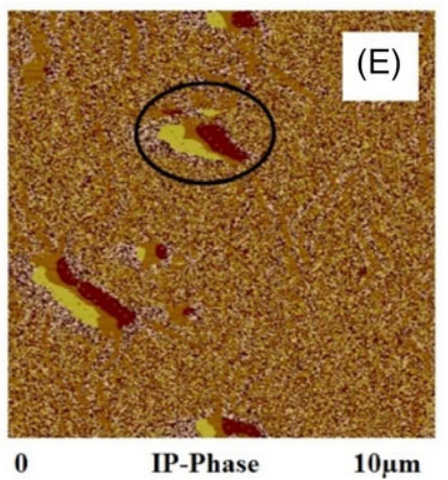

$180.0^{\circ}$

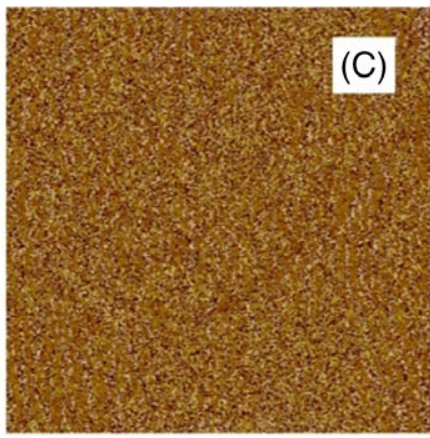

OP-Phase

$10 \mu \mathrm{m}$

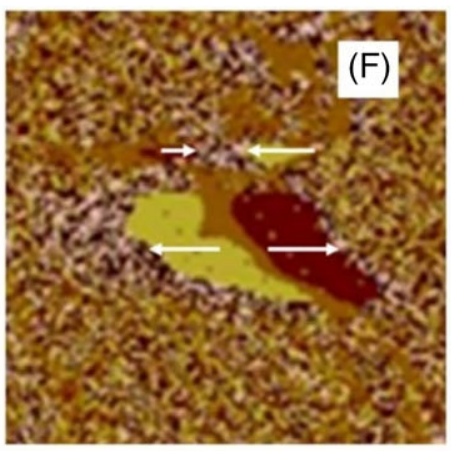

IP-Phase

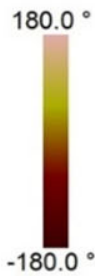

FIGURE 4 PFM images of PVDF films A, morphology (B,C), out of plane amplitude and phase images (D,E), in-plane (IP)amplitude and phase images $\mathrm{F}$, zoomed region of two $\mathrm{E}$, showing the nucleation of opposite orientations adjacent to the larger domains

\section{3 | Theoretical analysis}

We constructed checkerboard-type domain configurations similar to those in PFM images and implemented an inhomogeneous poisson solver to compute electric field and electrostatic energy density to study the effect of electrostatic interactions between the polar domains separated by a nonpolar medium. Further, these calculations are useful to estimate the equilibrium separation distances between different domain arrangements. Figure 5A shows one typical initial configuration of in-plane ferroelectric domains separated by a paraelectric matrix (red). Each pair of domains (denoted by yellow and
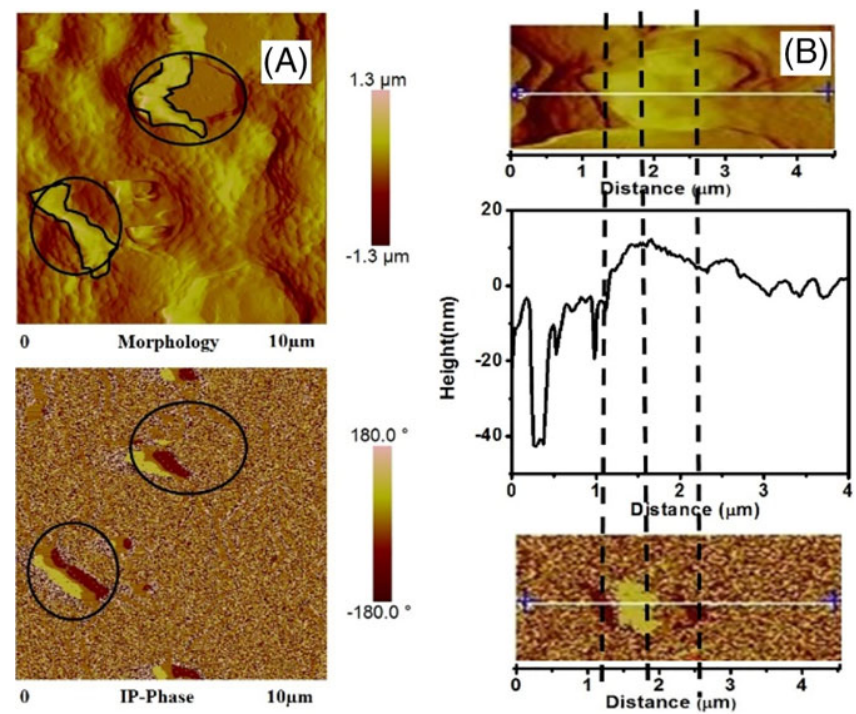

FIGURE 5 ( $A, B)$, Shows the influence of surface morphology on domain nucleation $\mathrm{A}$, for a $10 \mu \mathrm{m}$ region $\mathrm{B}$, for a particular domain black) in the checkerboard structure shares a $180^{\circ}$ domain wall. The separation distance along $x$-axis is kept constant while that along $y$-axis is varied systematically using a step size of $10 \mathrm{~nm}$. We compute the electrostatic energy of each configuration as a function of vertical separation distance. The minimum in electrostatic energy is used as a criterion to determine electrostatically most stable configuration. We used an average domain width of $600 \mathrm{~nm}$ and a horizontal separation distance of $\sim 250 \mathrm{~nm}$ to mimic experimentally observed domain patterns.

The electrostatic energy density and electric field distribution for a given configuration of domains is computed by solving the electrostatic equilibrium equation for electrically inhomogeneous systems ${ }^{[53]}$ :

$$
\nabla \cdot[\epsilon(\boldsymbol{r}) \nabla \varphi(\boldsymbol{r})]=\nabla \cdot \boldsymbol{P}(\boldsymbol{r}),
$$

where $\epsilon(\boldsymbol{r})$ is position-dependent dielectric permittivity, $\varphi(\boldsymbol{r})$ is the electric potential distribution and $P(r)$ denotes the inhomogeneous polarization field. ${ }^{[53]}$ The electric field is given as $E=-\nabla \varphi$ and the electrostatic energy density is given as

$$
F_{\text {electric }}=\frac{1}{2} \epsilon(\boldsymbol{r}) E^{2}+\boldsymbol{P} \cdot \mathbf{E}
$$

The electrostatic equilibrium equation was solved using INTEL MKL Library assuming periodic boundary conditions in absence of external field. ${ }^{[54]}$ Our calculations show a minimum electrostatic energy for the given configuration is minimum when the vertical separation distance varies between $350-450 \mathrm{~nm}$. Further increasing the distance increases the energy of the system (Figure $5 \mathrm{C}$ ). The good agreement between the experimental findings and numerical results indicate that arrangement of in-plane polar domains is primarily governed by the minimization of electrostatic interactions. Such a configuration is energetically stable because the amorphous layer 

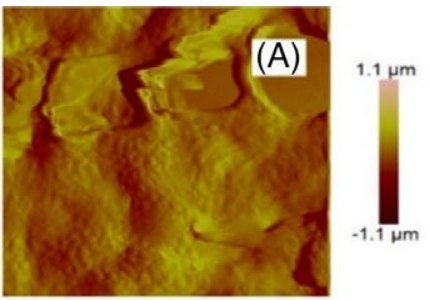

0 Morphology $10 \mu \mathrm{m}$
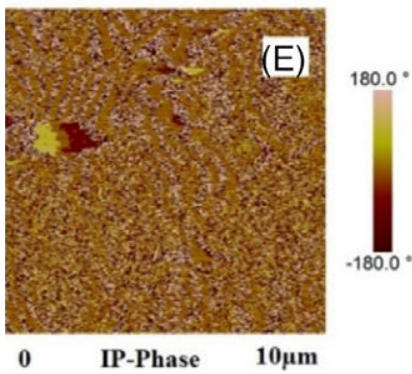
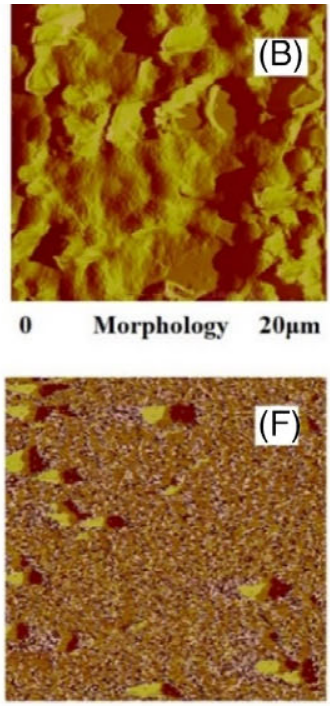

IP-Phase
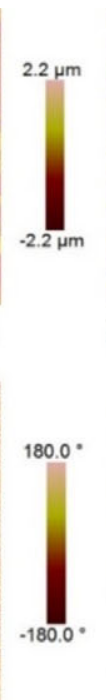

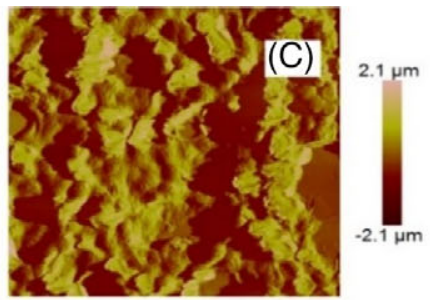

o Morphology

$30 \mu \mathrm{m}$

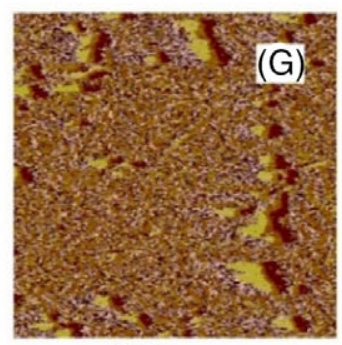

0

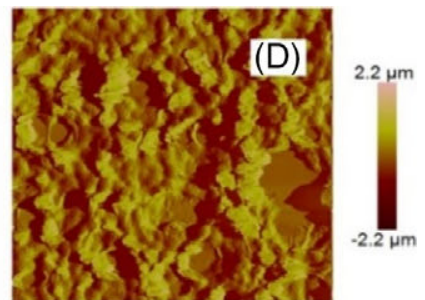

0 Morphology $40 \mu \mathrm{m}$

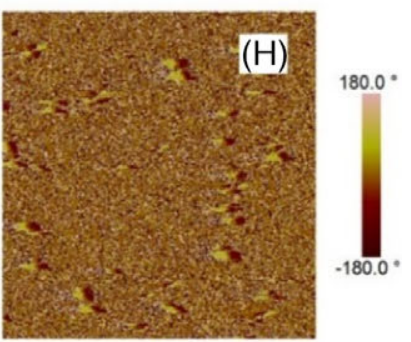

$0 \quad$ IP-Phase $40 \mu \mathrm{m}$

FIGURE 6 (A-D), Morphology of the scanned region (E-H), scalable distribution of domain across the scanned regions of PVDF film

(of 350-450 nm width) can effectively compensate the excess charge associated with the head-to-head/tail-to-tail configurations of the inplane domains when the separation distance is $\sim 390 \mathrm{~nm}$.) Most importantly, the domain size and separation distances obtained from the calculations corroborates well with the experimental observations. Figure 5D shows the electric field distribution in criss-cross domain configuration as shown in Figure 5A. In the central region (at the confluence of four domains), the electric field is almost fully compensated leading to very low electrostatic energy (Figure 5B). The $\beta$ phase of PVDF is thermodynamically meta-stable phase with all trans (TTTT) zigzag chain conformation. ${ }^{[13,44]}$ PVDF exhibits ferroelectric property as its electric dipole due to $\mathrm{C}-\mathrm{F}$ and $\mathrm{C}-\mathrm{H}$ can be changed in the presence of external electric field, ${ }^{[23]}$ which is confirmed by the ferroelectric hysteresis loop. The Switching spectroscopy (SS)-PFM studies performed in all the samples at various locations confirm the presence of ferroelectric hysteresis with switching characteristics. All the hysteresis loop in Figure 8 shows $180^{\circ}$ phase shift, Since the out-of-plane components contributes mainly to the hysteresis loop it is no surprise that our results seems to have weaker hysteresis as our films have shown dominating in-plane components. The PVDF 2.9 shows good hysteresis loop when compared to lower and higher viscous films. This can be attributed to increase in out-of-plane component with rise in viscosity.

\section{4 | Bias induced structural transformation}

Earlier studies suggest that application of electric field during the preparation of PVDF samples had its effect on the degree of
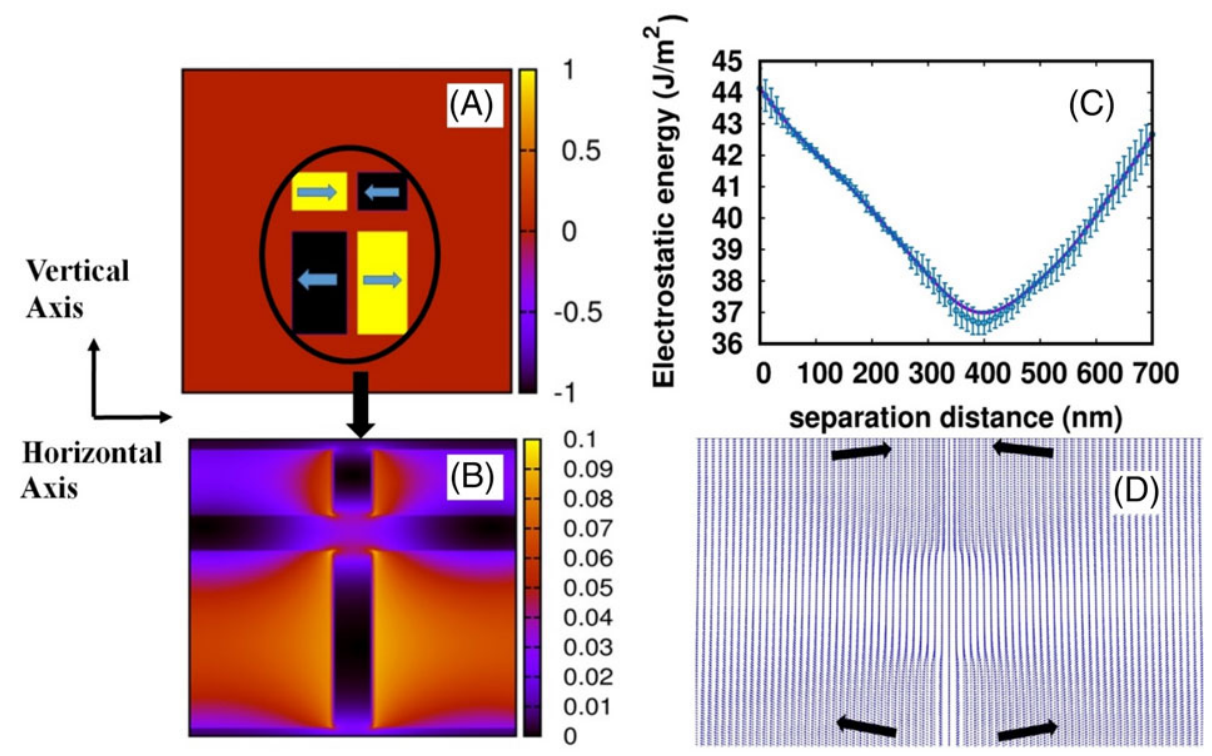

FIGURE 7 A, A typical configuration of the ferroelectric domains to compute electrostatic interactions between domains (similar to the domain arrangement observed in experiments). B, Electrostatic energy distribution when the vertical separation distance between the domains is $390 \mathrm{~nm} \mathrm{C}$, electrostatic energy vs vertical separation distance (along y-axis). D, Distribution of electric field in criss-cross domain configuration. The black arrows represent the direction of the electric field 

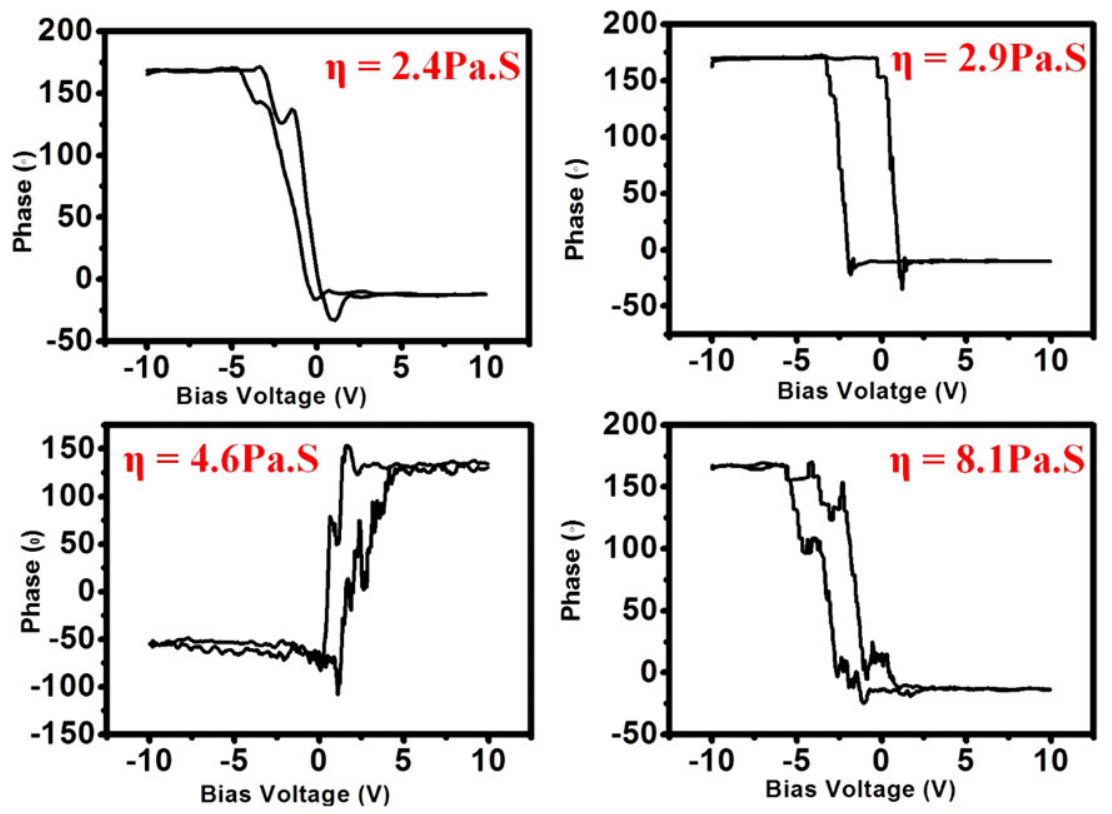

FIGURE 8 Piezoresponse force microscope (PFM) phase loop of PVDF film with different viscosities

crystallinity. ${ }^{[55]}$ It is known that the external electric field contributes in direction perpendicular to carbon backbone causing dipoles to rotate only with in-plane perpendicular to backbone. ${ }^{[45]}$ In this study on application of dc-bias across the PVDF thin films a variation in the domain pattern was observed which was similar to the domain patterns observed in completely crystalline PVDF-TrFE co-polymers. ${ }^{[46]}$ Figure 9A-F shows the PFM morphology, in-plane amplitude and inplane phase images for with and without bias. During PFM scan when the unpoled PVDF is subjected to sample bias $5 \mathrm{~V}$ and then further to
$10 \mathrm{~V}$, we observe there is a significant change and uniform distribution in the domain contrast which also indicates enhancement in the crystallinity of the film. The enhancement was prominent in the inplane compared to out-of-plane polarization. This growth is also visible in in-plane amplitude where the region of the domains undergoes similar change of shape and contrast growth. The electrostatic force within the PVDF during PFM alter the polymer chain causing monomer to rotate in the direction of electric field thus leading to change in the arrangement of irregular amorphous region and converting them

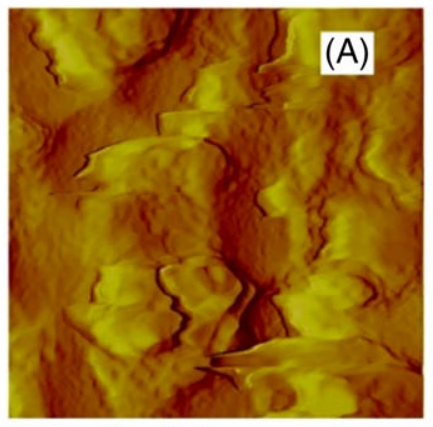

0
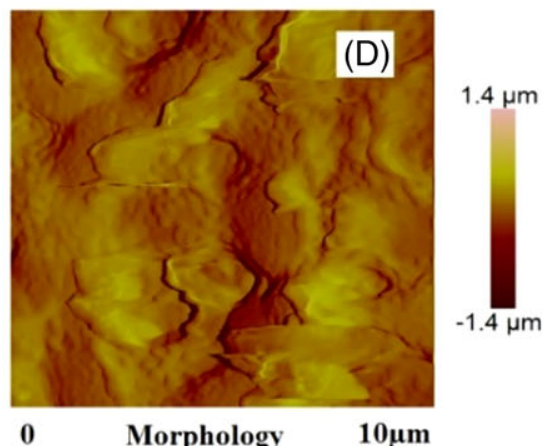

0

Morphology

$10 \mu \mathrm{m}$
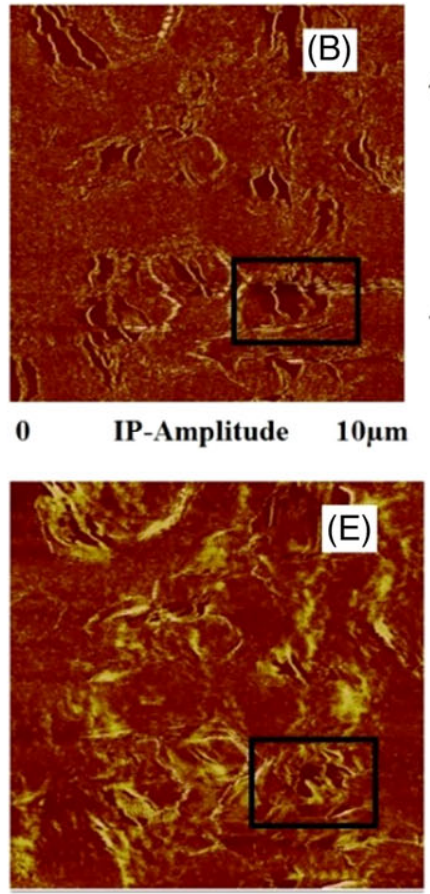

0

IP-Amplitude $\quad 10 \mu \mathrm{m}$
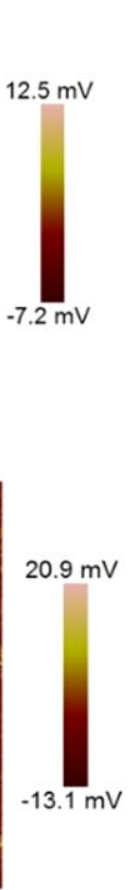
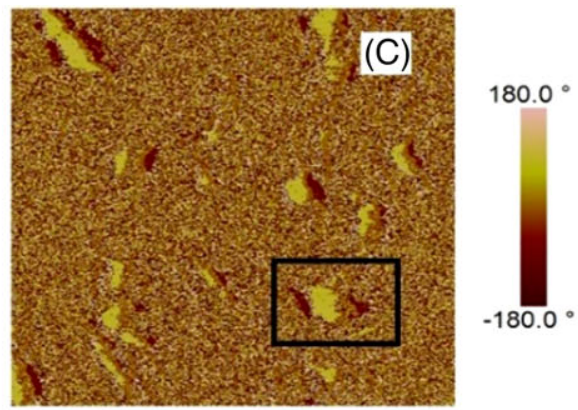

0

IP-Phase $\quad 10 \mu \mathrm{m}$

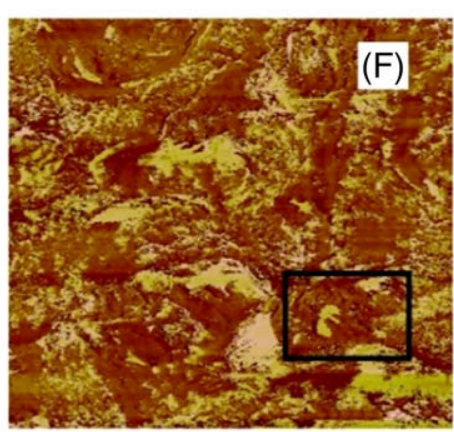

$180.0^{\circ}$

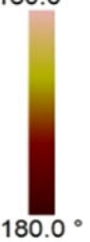

0

$10 \mu \mathrm{m}$

FIGURE 9 (A-C), Morphology, in-plane amplitude and phase of PVDF films under zero DC bias (D-F), morphology, in-plane amplitude, and phase of PVDF films induced under $5 \mathrm{~V}$ DC bias 


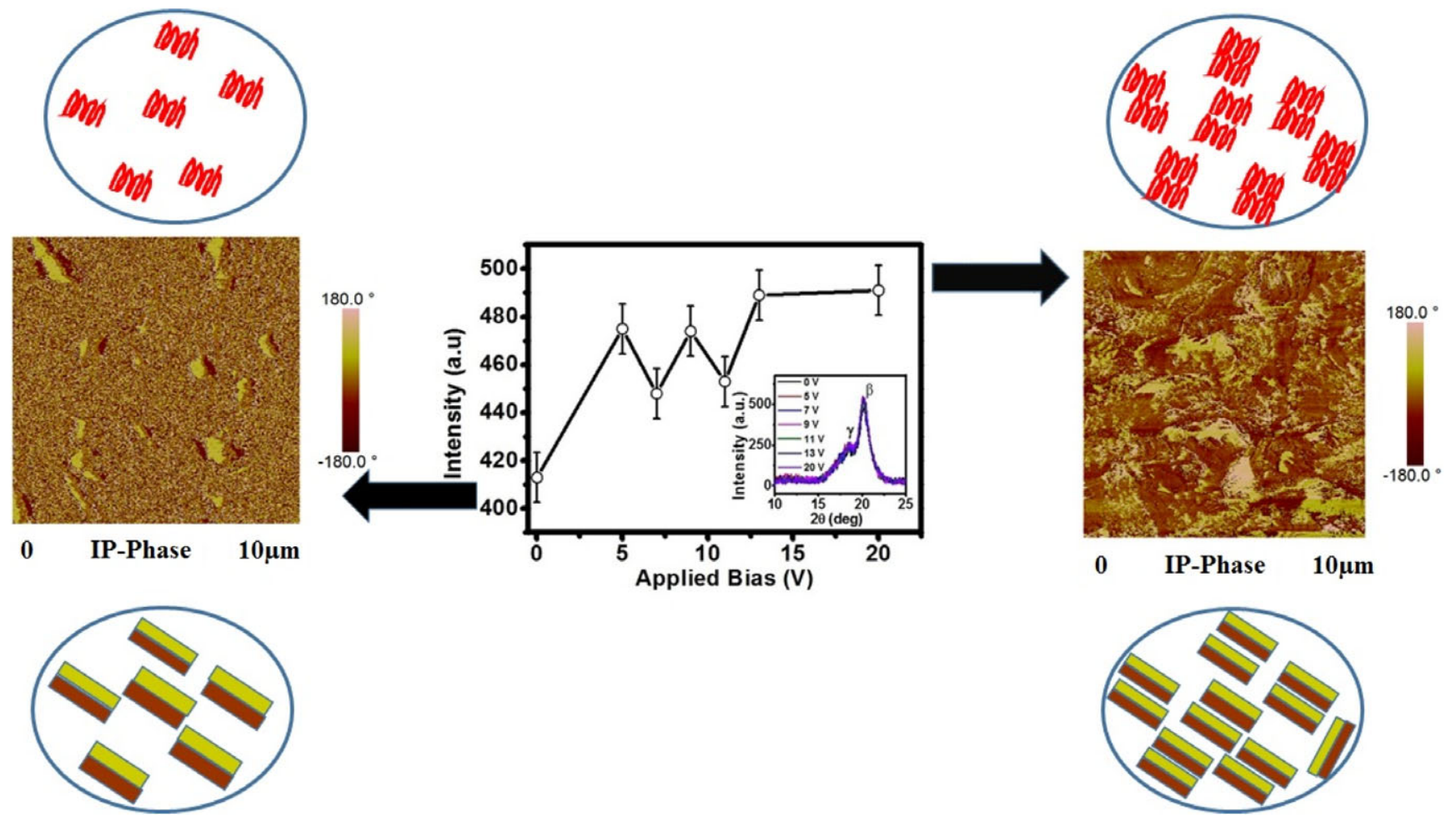

FIGURE 10 A, Variation of X-ray diffraction peak intensity of $\beta$-phase PVDF thin films obtained in the presence of DC bias B, schematic representation of polymer amorphous to crystalline transformation in the presence of DC bias

to a crystalline region. Literature has also suggested that in the presence of uniform electric field they observe increase in contrast in presence of dc bias which is similar our results. ${ }^{[23,27,56]}$ As Pure PVDF is expected to be semi-crystalline such a transformation of in-plane phase contrast in the domains is expected to be associated with an amorphous to crystalline transformation. In order to confirm such a transformation if any present, we performed the XRD studies in the presence of an applied electric field.

PVDF samples with thickness of $\sim 20 \mu \mathrm{m}$ on glass substrate with both platinum electrodes on top facilitating an applied field in planar configuration was fabricated. Sample was aligned carefully such that the $x$-ray scans only the region between the electrodes. XRD on the sample was performed at $0 \mathrm{~V}-20 \mathrm{~V}$ and the corresponding change was observed as a function of the peak intensity. As voltage increases from $0 \mathrm{~V}$ to $5 \mathrm{~V}$ a significant rise in $\beta$ phase peak intensity was observed. The rise was almost $15 \%$ when compared to the intensity obtained at $0 \mathrm{~V}$. The intensity oscillates around the same value between $7 \mathrm{~V}$ and $11 \mathrm{~V}$ and then a further increase in peak intensity is observed up to $20 \mathrm{~V}$ and saturates further. Figure 10 shows schematic representation of the correlation between the intensity rise and domain growth in association with amorphous to crystalline transformation. At $0 \mathrm{~V}$ the semi-crystalline polymer contains fewer crystalline regions (represented by chains structure) which are also indicated by the low peak intensity value in graph. As the voltage increases the peak intensity increases. This rise in peak intensity can be considered as increase in crystalline region and thereby increase in domain region. At voltage above $20 \mathrm{~V}$ when the intensity saturates it resembles that the maximum crystallinity for the film was obtained and further rise will not affect the amorphous to crystalline transformation. Previous studies showed similar enhancement in intensity when PVDF films were subjected to poling and later analyzed by $\mathrm{x}$-ray diffraction studies. ${ }^{[13,55]}$

In the present case we have studied the variation of intensity with an in-situ application of external bias and the variation of intensities are in good correlation with earlier studies. This increase in $\beta$ phase intensity of our PVDF films confirms the increase in degree of crystallinity under application of electric field. The $\gamma$ peak also follows the similar trend indicating the ratio of mixed phase remains unchanged across the transformation. Hence, the changes observed in the crystal structure due to electric field do not seem to affect the crystal phase of PVDF.

\section{4 | CONCLUSION}

In summary, we studied the polarization behavior of PVDF film over a viscosity range. The PVDF films exhibits a dominant planar domains with a unique pairing of domains. The amorphous region separating the domains is capable of holding excess charge and hence facilitates head-to-head or tail-to-tail kind of domain pair configuration in PVDF films. The electrostatic energy of such domain configuration was calculated and found to be in good correlation with the experimental observations. The influence of external bias leads to a change in domain configuration and size and is associated with an amorphous to crystalline transformation. Such a transformation was evidently observed in XRD studies in the presence of applied electric field. 


\section{5 | ACKNOWLEDGEMENT}

Mr. Ronit Ganguly is supported under the Visvesvaraya Ph.D. Scheme of the Ministry of Electronics and Information Technology (MEITY), Government of India (GOI). Dr. Amit Acharyya's research is supported by the "Visvesvaraya Young Faculty Fellowship Scheme" by MEITY, GOI".

\section{ORCID}

Ranjith Ramadurai (D) http://orcid.org/0000-0003-2991-0027

\section{REFERENCES}

[1] Z. Zhang, C. Yao, Y. Yu, Z. Hong, M. Zhi, X. Wang, Adv. Funct. Mater. 2016, 26, 6760 .

[2] T. Vuorinen, M. Zakrzewski, S. Rajala, D. Lupo, J. Vanhala, K. Palovuori, S. Tuukkanen, Adv. Funct. Mater. 2014, 24, 6340.

[3] S. K. Hwang, I. Bae, S. M. Cho, R. H. Kim, H. J. Jung, C. Park, Adv. Funct. Mater. 2013, 23, 5484.

[4] P. Martins, S. Lanceros-Méndez, Adv. Funct. Mater. 2013, 23, 3371.

[5] P. Martins, A. C. Lopes, S. Lanceros-Mendez, Prog. Polym. Sci. 2014 39, 683.

[6] S. M. Nakhmanson, M. B. Nardelli, J. Bernholc, Phys. Rev. Lett. 2004, 92, 115504.

[7] F. Liu, Q. Li, J. Cui, Z. Li, G. Yang, Y. Liu, L. Dong, C. Xiong, H. Wang, Q. Wang, Adv. Funct. Mater. 2017, 27, 1606292.

[8] W. J. Hu, D. M. Juo, L. You, J. Wang, Y. C. Chen, Y. H. Chu, T. Wu, Sci. Rep. 2014, 4, 4772.

[9] T. Prabhakaran, J. Hemalatha, Mater. Chem. Phys. 2013, 137, 781.

[10] A. J. Lovinger, Jpn. J. Appl. Phys. 1985, 24, 18.

[11] F. Chen, Y. Lu, X. Liu, J. Song, G. He, M. K. Tiwari, C. J. Carmalt, I. P. Parkin, Adv. Funct. Mater. 2017, 27, 1702926.

[12] G. M. Sessler, J. Acoust. Soc. Am. 1981, 70, 1596.

[13] J. Liu, X. Lu, C. Wu, Membranes 2013, 3, 389.

[14] S. Weinhold, M. H. Litt, J. B. Lando, Macromolecules 1980, 13, 1178.

[15] F. Liu, N. A. Hashim, Y. Liu, M. R. M. Abed, K. Li, J. Membr. Sci. 2011 $375,1$.

[16] M. Kim, J. K. Kim, J. H. Park, Adv. Funct. Mater. 2015, 25, 3399.

[17] R. Gregorio Jr., J. Appl. Polym. Sci. 2006, 100, 3272.

[18] S. Satapathy, S. Pawar, P. K. Gupta, K. B. R. Varma, Bull. Mater. Sci. 2011, 34, 727.

[19] V. Ranjan, M. B. Nardelli, J. Bernholc, Phys. Rev. Lett. 2012, 108, 087802.

[20] P. Sukitpaneenit, T. S. Chung, J. Membr. Sci. 2009, 340, 192.

[21] A. Bottino, G. Camera-Rodab, G. Capannelli, S. Munari, J. Membr. Sci. 1991, 57, 1.

[22] M. Benz, W. B. Euler, O. J. Gregory, Langmuir 2001, 17, 239.

[23] P. Sharma, D. Wu, S. Poddar, T. J. Reece, S. Ducharme, A. Gruverman, J.Appl. Phys. 2011, 110, 052010.

[24] M. G. Broadhurst, G. T. Davis, J. E. McKinney, J.Appl. Phys. 1978, 49, 4992.

[25] S. Liang, H. Yang, H. Yang, B. Tao, A. Djeffal, M. Chshiev, W. Huang, X. Li, A. Ferri, R. Desfeux, S. Mangin, D. Lacour, M. Hehn, O. Copie, K. Dumesnil, Y. Lu, Adv. Mater. 2016, 28, 10204.

[26] J. H. Yang, T. Ryu, Y. Lansac, Y. H. Jang, B. H. Lee, Org. Electron. 2016, 28, 67.

[27] P. S. Bednyakov, T. Sluka, A. K. Tagantsev, D. Damjanovic, N. Setter, Sci. Rep. 2015, 5, 15819.
[28] M. Poulsen, A. V. Sorokin, S. Adenwalla, S. Ducharme, V. M. Fridkin, J. Appl. Phys. 2008, 103, 034116.

[29] M. M. Costa, J. A. Giacometti, Appl. Phys. Lett. 1993, 62, 1091.

[30] K. Koga, H. Ohigashi, J.Appl. Phys. 1986, 59, 2142.

[31] P. Hu, Y. Shen, Y. Guan, X. Zhang, Y. Lin, Q. Zhang, C. W. Nan, Adv. Funct. Mater. 2014, 24, 3172.

[32] K. J. Kim, G. B. Kim, Polymer 1997, 38, 4881.

[33] K. Tashiro, Y. Itoh, M. Kobayashi, H. Tadokoro, Macromolecules 1985 18,2600

[34] G. Zhu, J. Xu, Z. Zeng, L. Zang, X. Yan, J. Li, Appl. Surf. Sci. 2006, 253 , 2498.

[35] A. Baji, Y. W. Mai, Q. Li, Y. Liu, Nanoscale 2011, 3, 3068.

[36] M. Sharma, V. Srinivas, G. Madras, S. Bose, RSC Adv. 2016, 6, 6251.

[37] A. Gebrekrstos, G. Madras, S. Bose, ACS Omega 2018, 3, 5317.

[38] D. Faust, R. Lakes, Ferroelectrics 2015, 481, 1.

[39] V. F. Cardoso, G. Minas, C. M. Costa, C. J. Tavares, S. Lanceros-Mendez, Smart Mater. Struct. 2011, 20, 087002.

[40] R. Magalhães, N. Durães, M. Silva, Silva, V. Sencadas, G. Botelho, J. L. Gómez Ribelles, S. Lanceros-Méndez, Soft Mater. 2011, 9, 1.

[41] V. F. Cardoso, A. R. Machado, V. C. Pinto, P. J. Sousa, G. Botelho, G. Minas, S. Lanceros-Méndez, J Polym Sci B 2016, 54, 1802.

[42] M. Kanik, O. Aktas, H. S. Sen, E. Durgun, M. Bayindir, ACS Nano 2014, 8, 99311.

[43] A. Hartono, S. Satira, M. Djamal, R. Ramli, H. Bahar, E. Sanjaya, Adv. Mater. Phys. Chem. 2013, 3, 71.

[44] J. Jones, L. Zhu, N. Tolk, R. Mu, Appl. Phys. Lett. 2013, 103, 072901.

[45] S. Chen, X. Li, K. Yao, F. E. H. Tay, A. Kumar, K. Zeng, Polymer 2012, $53,1404$.

[46] A. L. Kholkin, S. V. Kalinin, A. Roelofs, A. Gruverman, Scanning Probe Microscopy: Electrical and Electromechanical Phenomena at the Nanoscale, Springer, New York, USA 2007.

[47] E. Soergel, J. Phys. D Appl. Phys. 2011, 44, 464003.

[48] V. N. Kuleznev, V. A. Shershnev, The Chemistry and Physics of Polymers, Mir Publisher Moscow, Moscow, Russia 1990.

[49] V. N. Kuleznev, P. V. Surikov, Poly. Sci. 2012, 54, 833.

[50] G. Krause, D. Meurer, D. Klee, IEEE Trans. Electr. Insul. 1989, 24, 419.

[51] M. Fukuhara, T. Kuroda, F. Hasegawa, T. Sueyoshi, Sci. Rep. 2016, 6, 22012.

[52] L. Shaohui, Z. Jiwei, W. Jinwen, X. Shuangxi, Z. Wenqin, ACS Appl. Mater. Interfaces 2014, 6, 1533.

[53] J. J. Wang, X. Q. Ma, Q. Li, J. Britson, L. Q. Chen, Acta Mater. 2013, $61,7591$.

[54] INTEL MKL Library, https://software.intel.com (last updated: March 2017).

[55] W.G. Liu, X. Ying-xue, F. Hui-qing, Y. Chen, N. Xiao-ling, 18th ISAF, Xian, China, 23-27, 2009 August.

[56] B. J. Rodriguez, S. Jesse, S. V. Kalinin, Appl. Phys. Lett. 2007, 90, 122904.

How to cite this article: Ganguly R, Bandyopadhyay S, Miriyala $\mathrm{K}$, et al. Tunable polarization components and electric field induced crystallization in polyvinylidenefluoride: A piezo polymer. Polymer Crystallization. 2018;e10027. https://doi. org/10.1002/pcr2.10027 